\title{
The Digital Sky Survey of the Galactic Anti-center (DSS-GAC)
}

\author{
X.-W. Liu ${ }^{1,2}$, H.-B. Yuan ${ }^{1,3}$, Z.-Y. Huo ${ }^{4}$, M.-S. Xiang ${ }^{2}$, H.-H. Zhang ${ }^{2}$, \\ Y. Huang ${ }^{2}$, H.-W. Zhang ${ }^{2}$, H.-B. Zhao ${ }^{5}$, J. S. Yao ${ }^{5}$, H. Lu ${ }^{5}$ et al. \\ ${ }^{1}$ Kavli Institute for Astronomy and Astrophysics, Peking University, Beijing 100871, China \\ email: x.liu@pku.edu.cn \\ ${ }^{2}$ Department of Astronomy, Peking University, Beijing 100871, China ${ }^{3}$ LAMOST Fellow \\ ${ }^{4}$ National Astronomical Observatories, Chinese Academy of Sciences, Beijing 100012, China \\ ${ }^{5}$ Purple Mountain Observatory, Chinese Academy of Sciences, Nanjing 210008, China.
}

\begin{abstract}
As an integral component of the LAMOST Experiment for Galactic Understanding and Evolution (LEGUE; Deng et al. 2012), the LAMOST Galactic anti-center spectroscopic survey (Liu et al. in preparation) will survey over three thousand square degree sky area centered on the Galactic anti-center $(150 \mathrm{~d} \leqslant l \leqslant 210 \mathrm{~d},-30 \mathrm{~d} \leqslant b \leqslant+30 \mathrm{~d})$ and obtain low resolution $(R \sim 1800)$ optical spectra for a statistically complete sample of more than three million stars down to a limiting magnitude of 18.5 in $\mathrm{r}$ band, distributed in a spatially contiguous area and probing a significant volume of the Galactic thin/thick disks, halo and their interface. Sample stars of the LAMOST survey of the Galactic anti-center are derived from a recently completed CCD imaging photometric survey utilizing the newly built $1.0 / 1.2 \mathrm{~m}$ Schmidt Telescope at the Xuyi Station of the Purple Mountain Observatory. The Xuyi imaging survey (Yuan et al., in preparation; Zhang et al. 2012) provides high quality photometry ( $\sim 2$ per cent) in the SDSS g, $\mathrm{r}$ and $\mathrm{i}$ bands and astrometry $(\sim 0.1$ arcsec $)$ for about a hundred million stars down to a limiting magnitude of about 19 (10 sigma) for over six thousand square degree sky area ( $3 \mathrm{~h} \leqslant \mathrm{RA} \leqslant 9 \mathrm{~h}$, $-10 \mathrm{~d} \leqslant$ Dec $\leqslant+60 \mathrm{~d})$ that envelopes the LAMOST spectroscopic survey area of the Galactic anti-center, plus an extension to the M 31 and M 33 region.

This Digital Sky Survey of the Galactic Anti-center (DSS-GAC) with the Xuyi Schmidt and LAMOST telescopes will yield for the first time optical photometry and spectra for millions of stars in the Galactic disk(s), the defining component of the Milky Way as a typical spiral galaxy that contains most Galactic baryonic material and angular momentum. DSS-GAC will deliver classification, extinction, radial velocity and stellar parameters $\left(T_{\text {eff }}, \log g,[\mathrm{Fe} / \mathrm{H}]\right.$, probably also $[\alpha / \mathrm{Fe}]$, and in some cases, $[\mathrm{C} / \mathrm{Fe}])$, for each sample star. Together with the accurate proper motions and distances to be obtained with the forthcoming GAIA mission, DSS-GAC offers a unique opportunity for major breakthroughs in studies of the Galactic structure, formation and evolution. In particular, DSS-GAC will generate a huge data set to 1) study the stellar populations, chemical composition and kinematics of the thin and thick disks and their interface with the halo; 2) Understand how resilient galaxy disks are to gravitational interactions/perturbations and study the temporal and secular evolution of the disks; 3) identify tidal streams and debris of disrupted dwarfs and clusters; 4) probe the gravitational potential and dark matter distribution; 5) map the three-dimensional distribution and extinction of the interstellar medium; 6) search for rare objects (e.g. stars of peculiar chemical composition, hyper-velocity stars); and 7) ultimately advance our understanding of the formation and evolution of stars and galaxies.

Following a two-year commissioning, the LAMOST pilot survey was initiated in October, 2011 and completed in June, 2012. In total, about 370,000 spectra of 270,000 stars have been obtained for DSS-GAC, with 70 per cent of the spectra reaching a spectral S/N ratio per resolution element at $7150 \AA$ higher than 20. The formal LAMOST DSS-GAC survey will commence in September, 2012 , and is expected to complete in five years.
\end{abstract}

Keywords. Galaxy: disk, structure, formation, evolution, Surveys, LAMOST 